\title{
Microstructure Evolution in Cold-Rolled Pure Titanium: Modeling by the Three-Scale Crystal Plasticity Approach Accounting for Twinning

\begin{abstract}
KAROL FRYDRYCH (D) and KATARZYNA KOWALCZYK-GAJEWSKA @
A three-scale crystal plasticity model is applied to simulate microstructure evolution in hcp titanium subjected to cold rolling. Crystallographic texture and misorientation angle development, as an indicator of grain refinement, are studied. The impact of twinning activity on both phenomena is accounted for by combining the original three-scale formulation with the probabilistic twin-volume consistent (PTVC) reorientation scheme. The modeling results are compared with available experimental data. It is shown that the simulated textures are in accordance with the experimental measurements. The basic components of misorientation angle distribution, especially in the range of high angle boundaries, are also well reproduced.
\end{abstract}

https://doi.org/10.1007/s11661-018-4676-2

(c) The Author(s) 2018

\section{INTRODUCTION}

IT is well known that improvement of material properties can be achieved by tailoring the microstructure. The microstructure can be refined by advanced plastic deformation processes. The mechanisms leading to refinement are not yet fully described, in spite of the huge effort that has been put into modeling. While this statement is true in the case of face centered cubic (FCC) materials, it is even more relevant in the case of hexagonal close packed (HCP) materials. Mainly it is due to the fact that plastic deformation in $\mathrm{HCP}$ materials is usually more complicated than in FCC, because of the insufficient number of easy slip systems and the ease of twinning.

Qualitative studies of the grain refinement phenomenon in FCC metals and alloys were reported in References 1 through 7. Based on the indicated mechanisms of microstructure evolution, a three-scale crystal plasticity (3SCP) model was built. ${ }^{[8]}$ The model construction was motivated by the initially present deformation-induced cell substructure, schematically shown in Figure 1(a). Two types of grain boundaries can be recognized, namely geometrically necessary boundaries (GNBs) and incidental dislocation boundaries (IDBs). The new grains are formed by gradual increase of misorientation angles across the boundaries.

KAROL FRYDRYCH and KATARZYNA KOWALCZYKGAJEWSKA are with the Institute of Fundamental Technological Research (IPPT), Polish Academy of Sciences, Pawiǹnskiego 5B, 02-106 Warsaw, Poland. Contact e-mail: kfryd@ippt.pan.pl

Manuscript submitted January 11, 2018.

Article published online May 30, 2018
The mechanisms governing the grain refinement in metals of hexagonal close packed (HCP) lattice experiencing large plastic strains, induced e.g., via severe plastic deformation (SPD) processes, are known to a much lesser extent. First of all, in those metals, the number of easy slip systems is in general less than in FCC metals, and twinning appears as an additional, complementary mode. Second, in spite of the many efforts to carry out cold SPD processes for commercially pure titanium $(\mathrm{CP} \mathrm{Ti})^{[9]}$ and for AZ31b magnesium alloy, ${ }^{[10]}$ they are usually performed at elevated temperatures, which promote discontinuous dynamic recrystallization (DDRX). Beausir et al. ${ }^{[11]}$ assumed that the DRX for magnesium occurs at a temperature equal to $0.4 T_{\mathrm{M}}$, where $T_{\mathrm{M}}$ is the melting point $\left(T_{\mathrm{M}}=923 \mathrm{~K}\right.$ for magnesium). Thus $0.4 T_{\mathrm{M}}=369 \mathrm{~K}=96^{\circ} \mathrm{C}$, which is even less than the boiling point of water. Taking into account the simplicity of the $0.4 T_{\mathrm{M}}$ approximation and the fact that the temperature in the SPD process can increase without additional heating, it is not surprising that $\mathrm{Gu}$ et al. observed the DRX in AZ31b subjected to equal channel angular pressing (ECAP) process at room temperature. Figueiredo and Langdon ${ }^{[12]}$ proposed the model, in which DDRX is the mechanism responsible for grain refinement in the AZ31b alloy subjected to the ECAP process. New grains are formed near the initial grain boundaries or twin boundaries. The reason for this is the presence of higher stresses in those areas, which promote the activity of non-basal slip systems - in the case of sufficiently small grain (with size below the critical grain diameter), non-basal slip can activate throughout the whole volume of the grain. The critical value of the grain diameter can be dependent upon the given alloy, pressing temperature or the back-pressure used. Obtaining a homogeneous grain size distribution is 


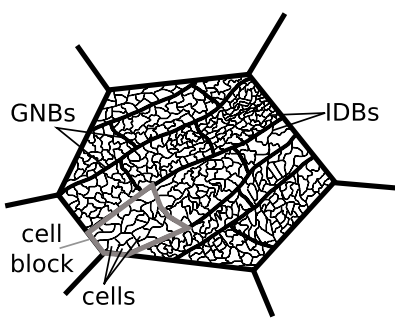

(a)

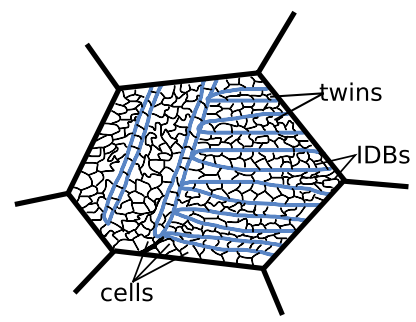

(b)
Fig. 1-(a) The schematic drawing of the dislocation-induced cell substructure of a grain observed in FCC materials for small to medium accumulated plastic strain according to Ref. [3], the picture reprinted after Ref. [8]. (b) The schematic showing analogous substructure for commercially pure titanium, after twinning.

thus feasible already in the first ECAP pass if the initial grain size is lower than the critical diameter. If the initial grain size is greater than the critical value, the microstructure obtained after the first pass is heterogeneous and contains both small and large grains. The model of Figueiredo and Langdon ${ }^{[12]}$ is consistent with many experimental data for AZ31b magnesium alloy, e.g., References 13 through 16.

For pure titanium, the melting point $T_{\mathrm{M}}$ is equal to $1941 \mathrm{~K}$; therefore, $0.4 T_{\mathrm{M}}=776 \mathrm{~K}=503{ }^{\circ} \mathrm{C}$, which is a much greater value than in the case of magnesium. According to the $0.4 T_{\mathrm{M}}$ criterion, the start of DRX is not expected in temperatures below $500{ }^{\circ} \mathrm{C}$, which justifies the approach excercised by some authors, ${ }^{[17,18]}$ who model the grain refinement of titanium assuming similar mechanisms to those acting in FCC materials. These works deal mainly with processes performed at low temperature (below $0.4 T_{\mathrm{M}}$ ). Yang et al. ${ }^{[17]}$ analyzed the grain refinement mechanism in shear bands that developed in cold-rolled CP Ti. They stated that the mechanisms responsible for grain refinement are analogous to those acting in FCC materials. On the other hand, twinning plays also an important role in the fragmentation of original grains. The authors noticed that the microstructure in shear bands is similar to the one observed in SPD processes. Taking this into account, the supposition was made that the same mechanisms can lead to grain refinement in SPD processes. Zeng et al. ${ }^{[18]}$ analyzed the grain refinement mechanisms acting during hot compression (temperature range 673 to $973 \mathrm{~K}$ ) of $\mathrm{CP}$ Ti extruded tube and observed that twinning has strong impact upon the grain refinement. At $723 \mathrm{~K}\left(53 \mathrm{~K}\right.$ lower than $\left.0.4 T_{\mathrm{M}}\right)$, the high angle grain boundaries (HAGBs) developed by two parallel processes: gradual increase of misorientation angle across the low angle boundary (LAB) - analogous mechanism to the one present in FCC metals, and by deformation of the twin boundaries, $c f$. Figure 1(b).

There is quite a large number of papers reporting modeling of grain refinement in FCC metals. Our recent paper ${ }^{[8]}$ shortly reviews the approaches used for modeling the grain refinement using the macroscopic phenomenological, ${ }^{[19,20]}$ the mean-field crystal plasticity models enhanced by some additional features ${ }^{[8,21-26]}$ and the incremental energy minimization-based models. ${ }^{[27-30]}$ Apart from the approaches discussed in Reference 8, models applying the crystal plasticity finite element method (CPFEM) with many integration points or elements per grain ${ }^{[31-35]}$ are also worth mentioning as a possible tool to predict the grain refinement. However, none of the models mentioned is able to fully predict the grain refinement in FCC materials.

As the mechanisms leading to grain refinement in HCP metals and alloys are by far less known than the analogous mechanisms for FCC materials, there are accordingly fewer papers modeling this phenomenon. As already mentioned, the grain refinement in AZ31b magnesium alloy is attributed to DDRX..$^{[12,13]}$ The simulation of grain refinement in an unconventional process joining extrusion and ECAP was described in Reference 36 . The process was performed at $350{ }^{\circ} \mathrm{C}$. The grain dimension after recrystallization was calculated using a phenomenological equation, calibrated for compression tests. The authors claimed that they had obtained an interesting agreement between the simulation and experimental data, but did not include direct comparison. The grain refinement stemming from DDRX can also be modeled using cellular automata (CA). Such an approach was applied by Gzyl et al. ${ }^{[37]}$ The strain, strain rate, and temperature at the integration points were calculated using the finite element method. These data were then used to simulate the DRX using the CA. The refinement in AZ31b subjected to ECAP and I-ECAP was simulated and good agreement between the results of modeling and experimental data was achieved.

Grain refinement in CP Ti subjected to SPD processes at only slightly elevated temperatures was modeled assuming mechanisms analogous to FCC materials. Ding and Shin ${ }^{[38]}$ classified the orthogonal cutting as a severe plastic deformation process, because of the ultrafine grains developing inside chips. The grain dimensions inside chips were predicted using a model of dislocation evolution ${ }^{[39,40]}$ inside dislocation cells and walls between them. A similar approach was applied in Reference 41 for modeling the grain refinement in cold rolling of titanium. The maximum temperature was determined to be $150{ }^{\circ} \mathrm{C}$. The average dislocation cell dimensions were predicted in good agreement with experimental results.

In the present paper, the texture and grain refinement of CP Ti deformed in cold rolling are studied. The evolution of microstructure of this material subjected to cold rolling was analyzed in References 42 through 46. In References 42, 44 through 46, only experimental data were analyzed. Bozzolo et al.$^{[33]}$ have modeled texture evolution during the process using the visco-plastic self-consistent (VPSC) ${ }^{[47]}$ model. The grain refinement was not simulated in any of those papers.

The authors of papers ${ }^{[42-46]}$ concluded that:

- Extension twinning systems of the family $\{10 \overline{1} 2\}\langle 10 \overline{1} 1\rangle$ (E1) and contraction twinning systems of the family $\{11 \overline{2} 2\}\langle 11 \overline{2} \overline{3}\rangle(\mathrm{C} 1)$ were activated.

- Activation of E1 twinning requires a lower resolved shear stress (RSS) than C1, but the RSS for most grains is initially much higher for the contraction 
twinning mode due to the strong initial texture. As a result, the $\mathrm{C} 1$ twin modes are activated first and the E1 modes are initiated mainly as a secondary twinning mode inside the $\mathrm{C} 1$ twins.

- Twinning is an important grain refinement mechanism, especially in the initial stages of deformation. Twin boundaries contribute to the fraction of high angle boundaries and the texture evolves due to the twin reorientation.

- For higher strains, the twinning saturates and then most of the deformation is accommodated by slip on the prismatic planes $\{10 \overline{1} 0\}$. The grain refinement continues by means of mechanisms analogous to those occurring in FCC metals.

The main goal of this paper is to verify to which extent the recently proposed computationally efficient three-scale crystal plasticity (3SCP) model ${ }^{[8,48]}$ is applicable to simulate texture evolution and grain refinement in titanium. To this aim, first, the 3SCP model is extended to account for twinning and next, the simulations of cold rolling are performed for $\mathrm{CP}$ titanium. In the proposed extension of the 3SCP model, the probabilistic twin-volume consistent (PTVC) reorientation scheme is used in order to properly account for the appearance of twin-related orientations at the metagrain level. Modeling results are analyzed and compared to experimental data available in the literature. The quality of model predictions is discussed and possible sources of discrepancies between simulations and experimental data are indicated.

The paper is organized as follows: After this introductory section, the 3SCP model formulation is recalled in Section II. First, the construction of a polycrystalline representative volume is outlined, and then the constitutive descriptions used on the lowest level of microstructure and rules of micro-macro transition are presented. The extension of the original framework due to twinning activity is also discussed. In Section III, the parameters of the crystal plasticity model are identified using the standard two-scale formulation, and in Section IV the three-scale approach is applied to predict the grain refinement and texture evolution in CP titanium subjected to cold rolling. The outcomes of simulations are compared with the experimental data available in the literature. ${ }^{[7]}$ Modeling results are discussed in detail in Section V. The paper is concluded in Section VI.

\section{MODEL OF GRAIN REFINEMENT}

The three-scale model ${ }^{[8]}$ applied in this paper to describe the microstructure evolution of HCP titanium was originally developed in order to enhance the texture predictions of the two-scale VPSC model ${ }^{[47]}$ and additionally predict the grain refinement in FCC materials by means of providing the misorientation angle distributions. In this section, the formulation of the model will be reviewed and its extension to model twinning will be presented.

The main difference between the classical two-scale model and the 3SCP approach is the additional subgrain level. Each grain is now called a metagrain and consists of a number of subgrains, $c f$. Figure 2. The notion of subgrain denotes a single discrete orientation and is not equal to the term subgrain in quantitative metallography, although it is conceptually related to it.

The representative volume element (RVE) in 3SCP model consists of $N G$ metagrains. In each metagrain, a nominal orientation $Q^{g}(0)$ is initially assigned. Metagrains are themselves aggregates of subgrains - each consists of NS subgrains, whose orientations are obtained by slightly distorting the nominal metagrain orientations. The initial orientation of subgrain $i$ is thus obtained as follows:

$$
Q_{i}^{g}(0)=R_{i}^{g}(0) Q^{g}(0),
$$

where

$$
R_{i}^{g}(0)=R\left(n_{i}, \delta \psi_{i}(0)\right)
$$

is a rotation matrix around arbitrary axis $n_{i}$ about the small angle $\delta \psi_{i}(0) \in\langle 0, \Delta \psi\rangle$. The axis $n_{i}$ and the distortion angle $\delta \psi_{i}$ are assigned to each subgrain by random generation.

During the simulation of some plastic deformation process, one can study the grain refinement occurring by evolution of subgrain orientations. The following equation describes the current misorientation angle $\delta \psi_{i j}^{g}(t)$ between subgrains $i$ and $j$ of the metagrain $g^{[49]}$ :

$$
2 \cos \left(\delta \psi_{i j}^{g}\right)=\operatorname{tr}\left(R_{i j}^{g}(t)\right)-1,
$$

where

$$
R_{i j}^{g}(t)=Q_{i}^{g}(t) Q_{j}^{g}(t)^{T} .
$$

Note that in Reference 8 , the misorientation angle was calculated with respect to the mean orientation of the metagrain. In this work, we have not used this approach in order to directly observe misorientation angles between matrix and twinned domains.

The original 3SCP model is extended to account for twinning by assuming relevant formulation of crystal plasticity theory at the lowest level of microstructure (i.e., subgrain level) and adopting the PTVC scheme for twin-related reorientation. The PTVC scheme ${ }^{[50]}$ accounts for lattice reorientation due to twinning. It preserves consistency of the reorientation probability with the current twin volume fraction in the grain, which results from twinning activity. The scheme originates from a statistical concept described in Reference 51. Detailed discussion concerning this condition can be found in Reference 52. Within the 3SCP model, the PTVC scheme is applied at the level of each subgrain, so if the reorientation condition is true a given subgrain within the metagrain is reoriented according to the most active twinning system. The PTVC scheme ensures that the volume fraction of twin-reoriented subgrains within the metagrain is consistent with the accumulated twin volume fraction in that metagrain predicted by the crystal plasticity model. Since the whole subgrain is reoriented instantaneously the scheme does not account for a growth of a twin inside the subgrain and development of the layered substructure. Such 


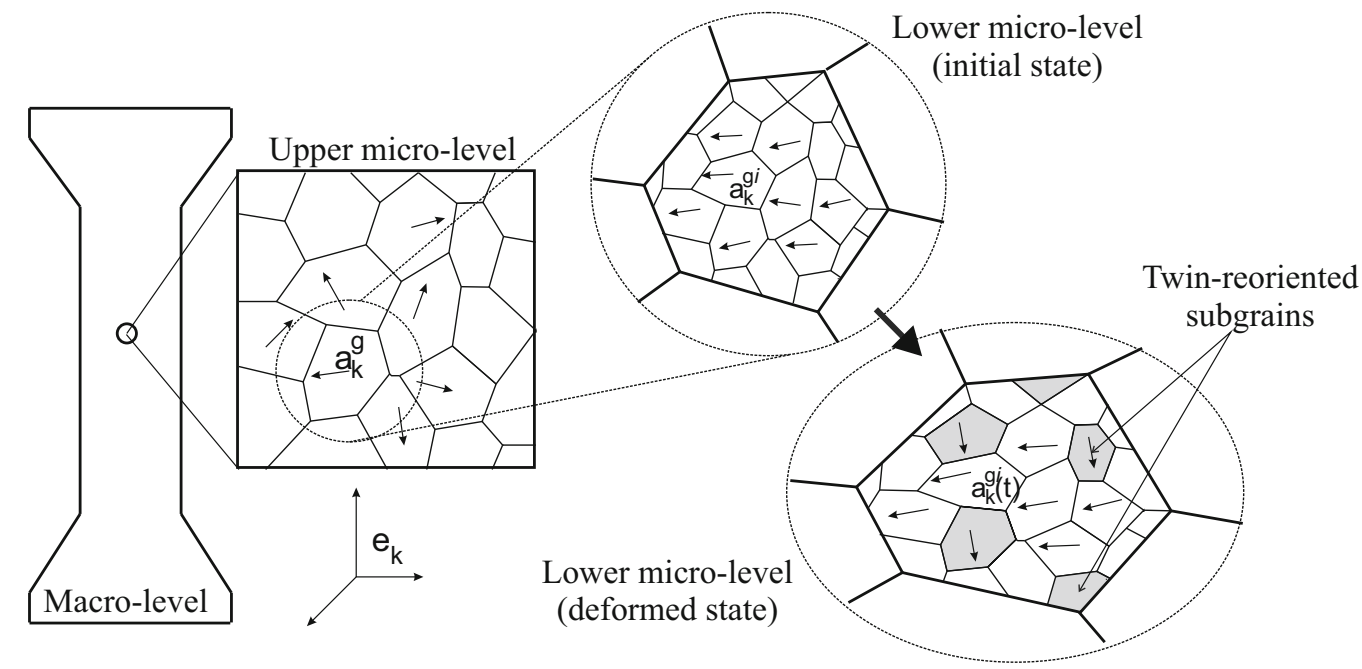

Fig. 2-Schematic view of the three-scale model.

morphological features are captured by models in which additional level of microstructure is introduced: (i) the total Lagrangian approach ${ }^{[33]}$ (ii) the composite grain model ${ }^{[54]}$ based on the formulae valid for laminates ${ }^{[55]}$ (iii) application of the ALAMEL model like in Reference 56, or (iv) variational energy-based model proposed in Reference 57. Extensive discussion on their capabilities can be found in Reference 52 . The crystal plasticity model with twinning used in the present study at the subgrain level is formulated within the Eulerian large-strain framework. The fundamentals of crystal plasticity theory can be found in References 58 and 59, and the details of twinning incorporation in References 50 and 60. The short summary of the approach is outlined below.

In terms of kinematics the additive decomposition of the velocity gradient $l$ into elastic and plastic parts is exploited. Elastic stretches are neglected (the elastic part $l^{e}$ is equal to the lattice spin), while the plastic part $l^{p}$ is specified as

$$
l^{p}=\sum_{k=1}^{2 M} \dot{\gamma}^{k} m^{k} \otimes n^{k}+\sum_{l=1}^{N} \dot{\gamma}^{l} m^{l} \otimes n^{l}, \text { where } \dot{\gamma}^{l}=\gamma^{T W} \dot{f}^{l},
$$

and $\dot{\gamma}^{k(l)} \geq 0$ is the rate of shearing on the $k(l)$ th slip (twin) system; $M$ and $N$ denote the number of slip and twin systems, respectively. Quantity $\dot{f}^{l}$ is the rate of volume fraction of twins that appeared due to activity of the twin system $l$, while $\gamma^{\mathrm{TW}}$ is the characteristic twin shear specified by the lattice geometry. Twinning is described as a unidirectional slip mode.

The classical viscoplastic power law

$$
\dot{\gamma}^{r}=\dot{\gamma}_{0}\left(\frac{\tau^{r}}{\tau_{c}^{r}}\right)^{n}
$$

relates the shear rate on the slip or twin system $r$ with the (non-negative) resolved shear stress $\tau^{r}=\left\langle m^{r} \cdot \boldsymbol{\sigma} \cdot n^{r}\right\rangle$ (where $\langle\cdot\rangle \equiv \frac{1}{2}((\cdot)+|\cdot|)$ ) and $\dot{\gamma}_{0}$ is the reference shear rate. The critical shear stresses $\tau_{c}^{r}$ evolve during plastic deformation according to the law summarized in Table I. The physical background for the applied formulation is extensively discussed in Reference 61.

Additionally, the fact that mechanical properties of the twins are modified with respect to the matrix ${ }^{[62]}$ is taken into account. A scale factor $\mu$ is introduced for slip and twin systems, and the critical shear stresses for the (sub)grain reoriented by PTVC scheme are changed to $\mu \tau_{c}^{0}$ where $\tau_{c}^{0}$ is the initial value of $\tau_{c}$. The quantities $\tau_{c}^{0}, \beta, \tau_{\text {sat }}, f_{\text {sat }}, \mu, q^{(\alpha \beta)}$ are material parameters to be determined by some identification procedure.

Two micromechanical schemes, related to the particular mean-field formulation, have to be selected for the scale transition between the subgrain-metagrain and metagrain-polycrystal levels. In Reference 8, the influence of the choice of mean-field models on the predictions of misorientation evolution were studied. Here, taking into account the results of the mentioned study, the Taylor model ${ }^{[63]}$ is applied for the transition between grain aggregate and metagrain levels, and the tangent variant $t^{47,64]}$ of the VPSC scheme ${ }^{[47,65]}$ for the transition between the metagrain and subgrain levels. The choice of the Taylor model for the higher micro-macro transition, and the VPSC for the lower can be supported as follows: Within the three-scale model, the subgrain is primarily interacting with its close vicinity, represented in the self-consistent model by the averaged metagrain behavior, and much less intensively with the whole polycrystal. The averaged metagrain behavior calculated in the VPSC model is continuously modified with increasing misorientation angles between subgrains and their twin-related reorientation. The iso-strain Taylor model disables strain redistribution between metagrains, thus increases strain heterogeneity between subgrains, and the resulting misorientation angle, enabled by the VPSC scheme. Use of the VPSC scheme for both scale transitions would decrease strain heterogeneity and the resulting misorientation between subgrains because the strain redistribution would take place in two stages. Better accordance with experimental 
data is observed for the first selection of scale-transition schemes. Additionally, the Taylor scheme allows for easy parallelization of computation. Thus, in order for the 3SCP model to be effective, the proposed choice of mean-field models is used.

It is worth mentioning that the proposed three-scale formulation can be easily, at least conceptually, extended to the multi-scale framework. This could be done by introducing some threshold value for misorientation angle upon which the subgrain is subdivided into domains with orientations slightly disturbed with respect to the current orientation of parent subgrain. The same procedure can be applied when the subgrain is reoriented due to twinning. Nevertheless, such subdivisions would lead to the excessively increased computational cost and have been postponed in the present analysis.

\section{SELECTION OF MODEL PARAMETERS}

In order to perform the simulation using the $3 \mathrm{SCP}$ model, it is first necessary to identify a set of material parameters for the single crystal model. In the present study, the parameters were obtained using the tangent variant of the two-scale VPSC model. The parameters were selected so as to predict correct texture in CP Ti sheet subjected to cold rolling, corresponding to the experimental ones presented in References 42 and 44. Since the stress-strain response is not on the focus of this paper, therefore the value of $\tau_{c 0}$ for prismatic slip was set to 1 . For the detailed prediction of stress-strain response, the additional verification of parameters should be performed. The parameters are shown in Table II. It is assumed that initially grains have spherical shape and the shape evolves to ellipsoidal due to the deformation.

Figure 3 shows the slip and twin systems activities in the cold rolling simulation. The most active slip system is the prismatic one, which is justified by its lowest critical resolved shear stress (CRSS) and texture favorable for the activity of the prismatic slip family. At the beginning, the second most active system was $\{11 \overline{2} 2\}\langle 11 \overline{2} \overline{3}\rangle$ twinning

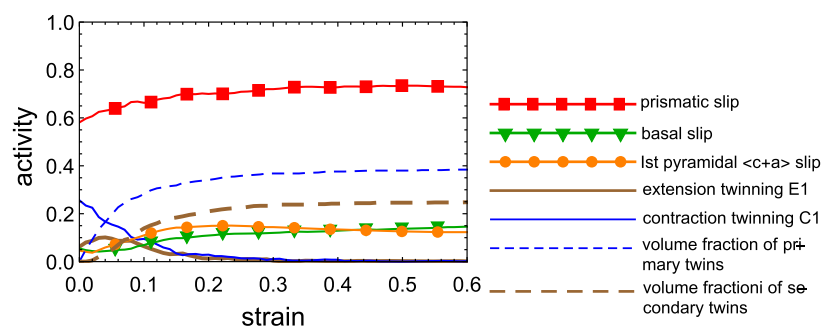

Fig. 3-Slip and twin systems activities obtained in the cold rolling simulation using the VPSC model for the selected set of parameters (Color figure online).

Table I. Summary of Hardening Description within the Applied Crystal Plasticity Model

Hardening Law with Four Types of Interactions

Slip-Slip $(s s)$, Slip-Twin $(s t)$, Twin-Slip $(t s)$, and Twin-Twin $(t t)$

For Slip $(r \leq M)$

For Twinning $(r>2 M)$

Hardening Moduli

Slip-Slip

Slip-Twin

Twin-Slip

Slip-Twin

Latent Hardening Submatrices

$$
\begin{aligned}
& \dot{\tau}_{c}^{r}=\dot{\tau}_{c}^{r+M}=H_{(s s)}^{r} \sum_{q=1}^{M} h_{r q}^{(s s)} \dot{\bar{\gamma}}^{q}+H_{(s t)}^{r} \sum_{q=2 M+1}^{2 M+N} h_{r q}^{(s t)} \dot{\gamma}^{q}, \text { where } \dot{\bar{\gamma}}^{q}=\dot{\gamma}^{q}+\dot{\gamma}^{q+M} \\
& \dot{\tau}_{c}^{r}=H_{(t s)}^{r} \sum_{q=1}^{M} h_{r q}^{(t s)} \dot{\bar{\gamma}}^{q}+H_{(t t)}^{r} \sum_{q=2 M+1}^{2 M+N} h_{r q}^{(t t)} \dot{\gamma}^{q} \\
& H_{(\alpha s)}^{r}=h_{0}^{\alpha s}\left(1-\frac{\tau_{c}^{r}}{\tau_{s a t}^{r}}\right)^{\beta} \\
& H_{(\alpha t)}^{r}=\frac{h_{0}^{\alpha t}}{\tau_{c}^{r}}\left(\frac{f^{T W}}{f_{s a t}^{t}-f^{T W}}\right) \\
& h_{r q}^{(\alpha \beta)}=q^{(\alpha \beta)}+\left(1-q^{(\alpha \beta)}\right)\left|n^{r} \cdot n^{q}\right|
\end{aligned}
$$

\begin{tabular}{|c|c|c|c|c|c|c|c|c|c|}
\hline \multirow[b]{2}{*}{ System } & \multirow[b]{2}{*}{ Interaction } & \multirow[b]{2}{*}{$\tau_{c 0}(\mathrm{MPa})$} & \multirow[b]{2}{*}{$h_{0}(\mathrm{MPa})$} & \multirow[b]{2}{*}{$\beta$} & \multirow[b]{2}{*}{$\tau_{\text {sat }} / f_{\text {sat }}(\mathrm{MPa}) /-$} & \multirow[b]{2}{*}{$\mu(-)$} & \multicolumn{3}{|c|}{$q$} \\
\hline & & & & & & & Prism. & $\begin{array}{l}\text { Basal } \\
\text { Twin }\end{array}$ & I Pyr $\langle c+a\rangle$ \\
\hline \multirow[t]{2}{*}{ Prism. } & slip-slip & 1.00 & 1.79 & 1 & 1.14 & 1 & 1.0 & 1.0 & 1.0 \\
\hline & slip-twin & - & 0.00 & - & 0.01 & - & & 2.0 & \\
\hline \multirow[t]{2}{*}{ Basal } & slip-slip & 2.94 & 0.01 & 1 & 14.29 & 2 & 1.8 & 1.8 & 1.8 \\
\hline & slip-twin & - & 0.01 & - & 0.01 & - & & 2.0 & \\
\hline \multirow{2}{*}{$\mathrm{I} \operatorname{Pyr}\langle c+a\rangle$} & slip-slip & 2.94 & 0.01 & 1 & 14.29 & 2 & 1.8 & 1.8 & 1.8 \\
\hline & slip-twin & - & 0.01 & - & 0.01 & - & & 2.0 & \\
\hline \multirow[t]{2}{*}{ E1 } & twin-slip & - & 0.07 & 1 & 0.01 & - & 1.0 & 1.0 & 1.0 \\
\hline & twin-twin & 1.41 & 0.01 & - & 0.00 & 1 & & 1.0 & \\
\hline \multirow[t]{2}{*}{$\mathrm{C} 1$} & twin-slip & - & 0.07 & 1 & 0.01 & - & 1.0 & 1.0 & 1.0 \\
\hline & twin-twin & 2.00 & 0.01 & - & 0.00 & 1 & & 1.0 & \\
\hline
\end{tabular}

For the physical background and further details see Ref. [61].

Table II. Single Crystal Plasticity Parameters Used in the Paper

Abbreviations: prism.: prismatic, I pyr. $\langle c+a\rangle$ : first-order pyramidal $\langle c+a\rangle$, E1: $\{10 \overline{1} 2\}\langle 10 \overline{1} 1\rangle$ extension twinning, $\mathrm{C} 1:\{11 \overline{2} 2\}\langle 11 \overline{2} \overline{3}\rangle$ contraction twinning. 
(C1), which was activated despite its relatively high CRSS, because of the favorable texture. Subsequently, the E1 twinning was activated, mainly as a secondary twinning in primary $\mathrm{C} 1$ twins. After the saturation of twinning activity, the deformation proceeded further by prismatic slip supported by basal and pyramidal ones. The predicted scenario is in accordance with the conclusions of References 42, 44 through 46 reported in the introduction. According to References 42 and 44, in the present case twinning saturates at higher thickness reductions because the grain size has become so small that further twinning is impossible. In the simulations, since the model does not explicitly account for the grain size, the volume fraction of twinning $f^{T W}$ (see Table II) at which it saturates is an additional model parameter $f_{\text {sat }}$. The function of hardening modulus $H_{t t}$ for the critical shear stress for twinning is formulated in a way which accounts for the geometrical effect of twin boundaries in reducing the mean free path distance. This formulation follows earlier work. ${ }^{[66]}$

Figure 4 shows $\{0001\},\{10 \overline{1} 0\}$ and $\{11 \overline{2} 0\}$ pole figures of (a) initial and (b) final texture in the VPSC simulation of cold rolling. The textures are demonstrated using the convention in which y-axis of an orthogonal crystal frame is coaxial with $a_{k}$ axes of hexagon, $c f$. Appendix. Such convention was chosen in order to enable the direct comparison with experimental data presented in References 42 through 46.

\section{SIMULATION OF MICROSTRUCTURE EVOLUTION OF COLD-ROLLED PURE TITANIUM}

Then, the 3SCP model was applied in order to simulate the microstructure evolution in CP Ti subjected to cold rolling. Equivalently with a two-scale model, an initial shape of subgrains is spherical and evolves to ellipsoidal due to the deformation. Deformation in the rolling process was approximated imposing the following macroscopic velocity gradient:

$$
L=\left[\begin{array}{ccc}
1 & 0 & 0 \\
0 & 0 & 0 \\
0 & 0 & -1
\end{array}\right]
$$

where the directions of axes 1, 2, 3 are rolling direction (RD), transverse direction (TD), and normal direction (ND), respectively.

The additional 3SCP model parameters were set to $n=10$ and $\Delta \Psi=0.3 \mathrm{deg}$. Figures 5 and 6 show the pole figures obtained in the simulation. Using the FC-Tg variant of the $3 \mathrm{SCP}$ model, it was possible to correctly predict the final texture obtained experimentally, $c f$. Reference 42-Figures 6 and 9, Reference 43-Figure 1, Reference 44-Figure 8 and Reference 45-Figure 5. In particular, it was possible to predict two "c" fibers inclined from the normal to the sheet plane ( $\{0001\}$ pole figure). It was also possible to predict the positions of maxima on $\{10 \overline{1} 0\}$ and $\{11 \overline{2} 0\}$ pole figures. The temporary existence of the " $\mathrm{c}$ " fiber normal to the sheet plane observed in References 42 through 44 was, however, not predicted in the $3 \mathrm{SCP}$ simulation. In Reference 43, the presence of the fiber was attributed to multiple twinning reorientations. On the other hand, this fiber was not observed in Reference 45. As shown in Figure 7, as compared to the two-scale VPSC model (Figure 3), in the three-scale model an increased activity of the hard pyramidal and basal modes at the cost of easy prismatic slip is observed in the twin-reoriented crystallites. This change is due to the use of the Taylor transition scheme at the upper level and seems to be the main source of difference in the texture image resulting from two simulations.
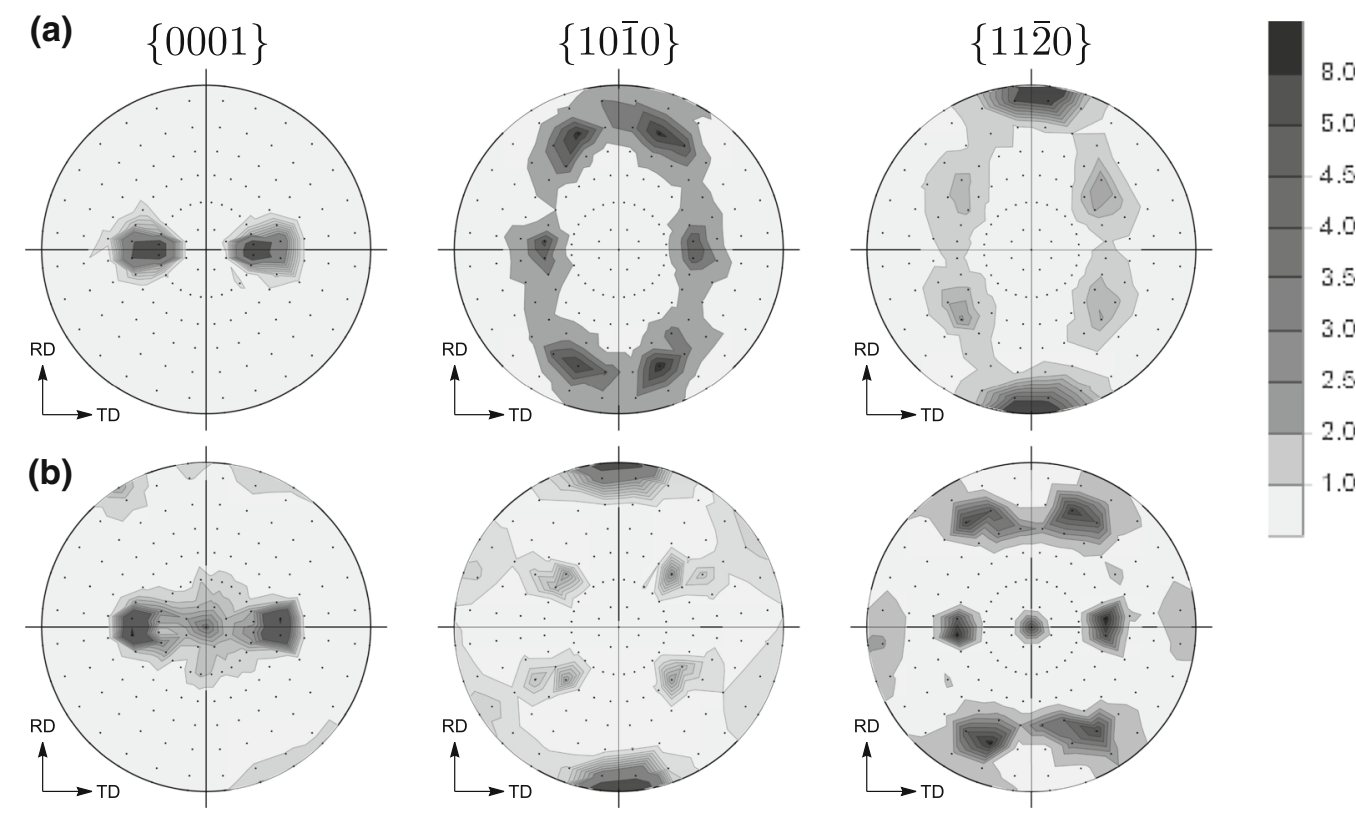

Fig. $4-\{0001\},\{10 \overline{1} 0\}$ and $\{11 \overline{2} 0\}$ pole figures of $(a)$ initial and $(b)$ final texture in the VPSC simulation of cold rolling to a total thickness reduction of 90 pct. 

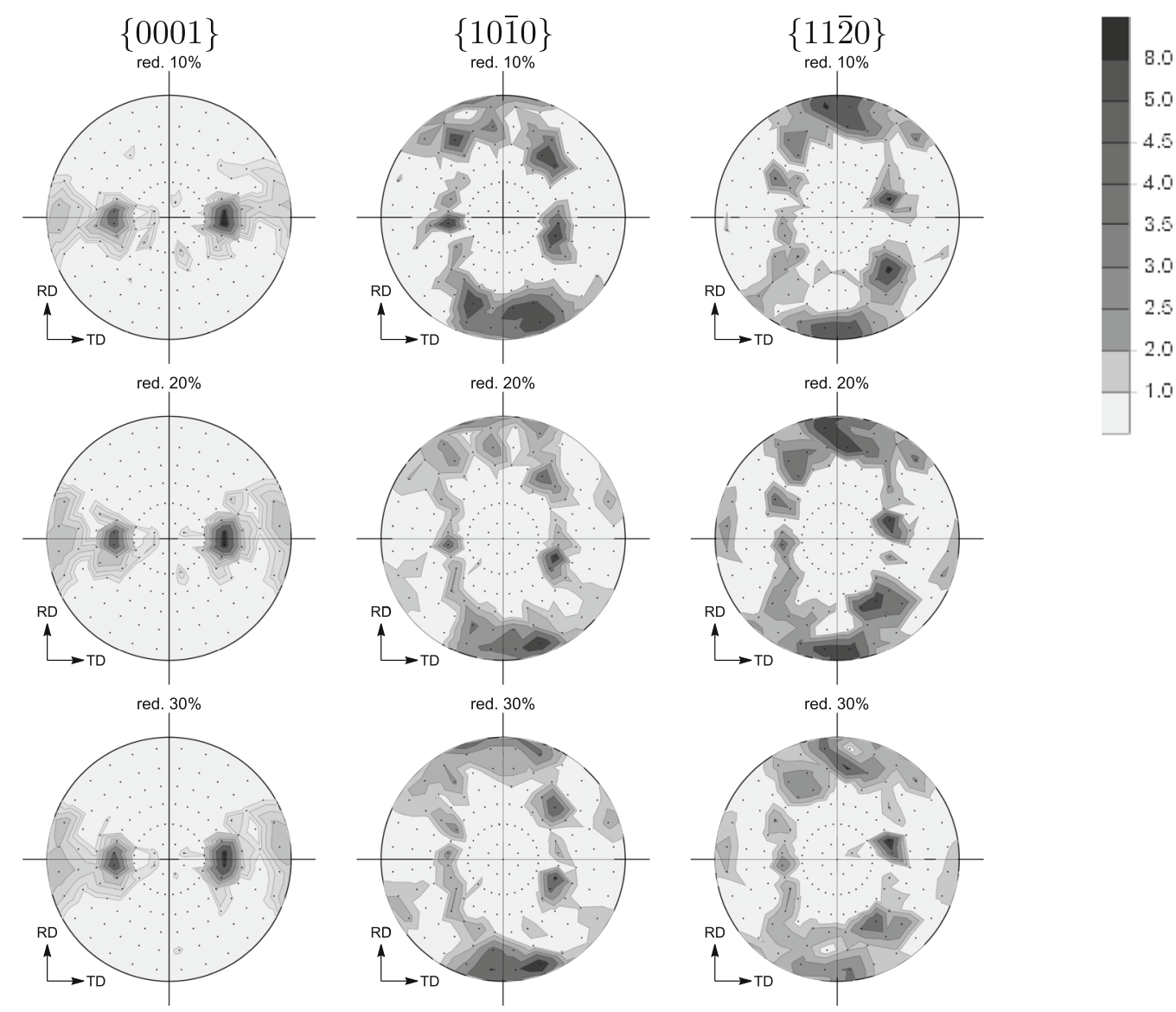

Fig. 5- $\{0001\},\{10 \overline{1} 0\}$ and $\{11 \overline{2} 0\}$ pole figures obtained in the 3SCP model simulation of cold rolling to a total thickness reduction of 10 , 20, and 30 pet.

Figure 8 shows plots of misorientation angle distribution obtained experimentally by Chun et al. ${ }^{[42]}$ and calculated using the results of 3SCP model simulation. Figure 9 presents the calculated misorientation-axis distribution for the strain reduction 20 and 90 pct, correspondingly. The latter results can be compared with Figure 8 in Reference 67. The simulated distributions were obtained by calculating the misorientation angles and axes between randomly selected pairs of subgrains in each metagrain. The randomly chosen pairs of subgrains are intended to mimic the neighboring areas present in real material. For any subgrain, a number of neighbors can be randomly selected. This number was assessed to be 12; therefore first, the misorientation distributions were calculated for misorientation angles between each subgrain and its randomly selected 12 "neighbors." In order to increase the numerical efficiency of the analysis, in the second step for each subgrain only one "neighbor" was randomly chosen. The misorientation angle distribution obtained using these two approaches was very similar ( $c f$. Figure 10); therefore, the results presented in the paper were obtained for the case of one neighbor per each subgrain. Column $b$ presents distributions obtained for every pair of "neighbors." Two maxima present in the experiment, namely for 64.4 and $85.0 \mathrm{deg}$, were qualitatively predicted in the simulation. The corresponding misorientation axes are oriented along $\langle 10 \overline{1} 0\rangle$ and $\langle 11 \overline{2} 0\rangle$ directions. According to Reference 42, and to our calculations, they correspond to the activity of twinning modes $\mathrm{C} 1$ and $\mathrm{E} 1$, respectively. They represent the misorientation angle at the boundary between primary $\mathrm{C} 1$ twin and secondary E1 twin (64.4 deg) as well as between the matrix and primary $\mathrm{C} 1$ twin $(85.0$ deg). The simulation predicts also additional maximum around $48.4 \mathrm{deg}$. It can be verified that this value represents misorientation angle between the secondary E1 twin and the matrix. It seems that this peak is not present in the experimental distributions, in which weighted length of the boundary is used, ${ }^{[67]}$ because the number and length of boundaries between secondary twins and matrix are very small, $c f$. Figure 11. Therefore, another misorientation angle distributions were calculated and are shown in the c column. This time the misorientation angles between secondary twins and matrix were not taken into account. There is qualitative agreement between the position and relative strength of the peaks obtained in the simulation and experiment. On the other hand in the misorientation-axis distribution plots, poles corresponding to matrix-secondary twin boundaries are seen in Reference 67 (see Figure 8 therein), in which a discrete point corresponds to a single boundary independently of its length. Thus, the calculated misorientation-axis distributions shown in 

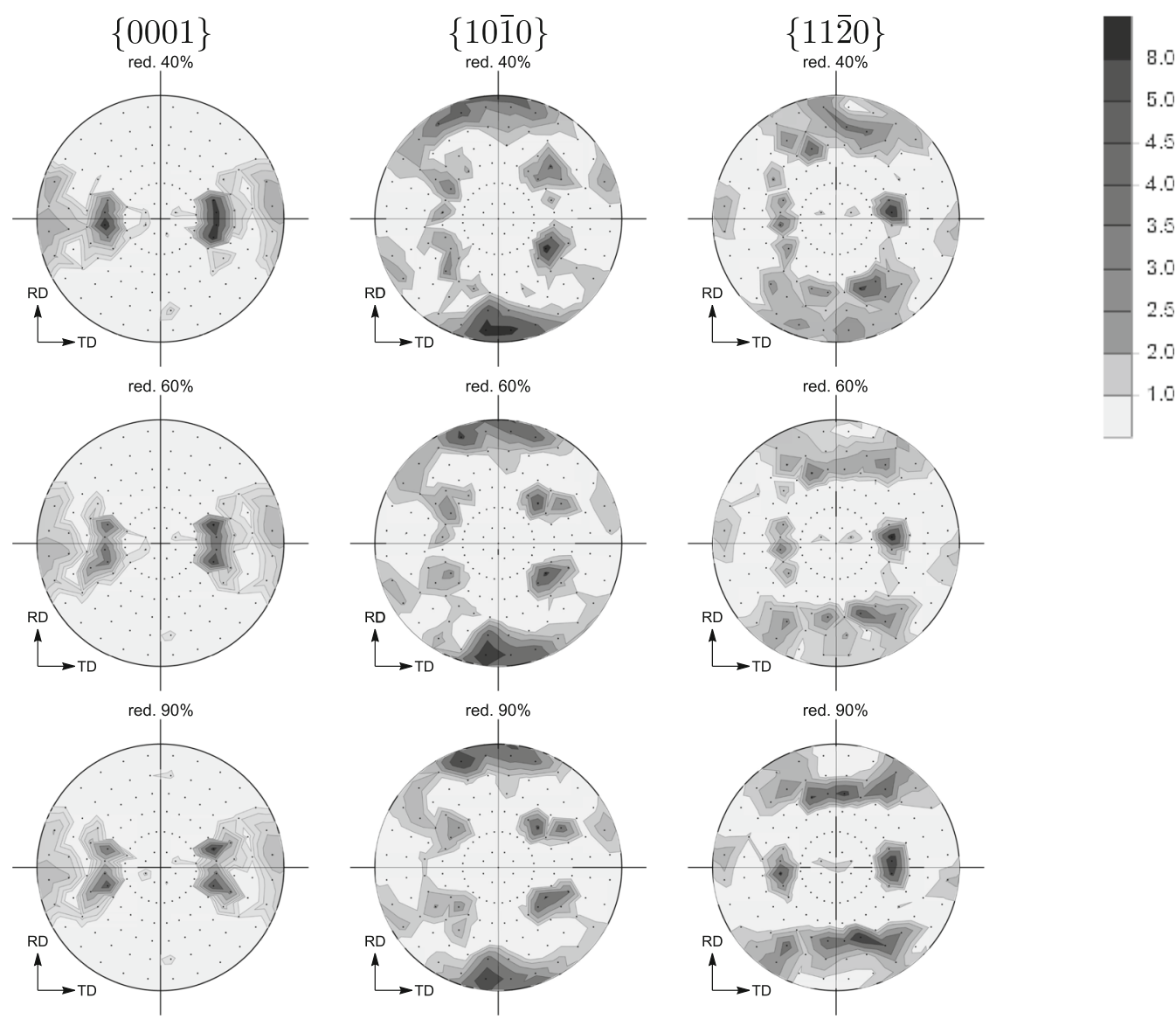

Fig. $6-\{0001\},\{10 \overline{1} 0\}$ and $\{11 \overline{2} 0\}$ pole figures obtained in the 3SCP model simulation of cold rolling to a total thickness reduction of 40 , 60 , and 90 pet.

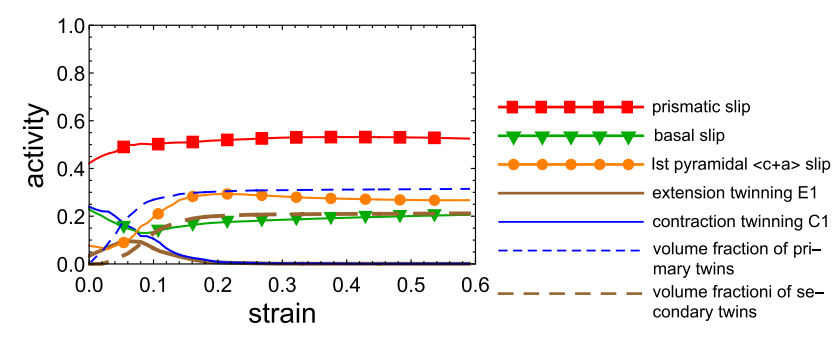

Fig. 7-Slip and twin systems activities obtained in the cold rolling simulation using the 3SCP model (Color figure online).

Figure 9 are presented for the case when the boundaries between the pairs composed of subgrain subject to the secondary twinning and the untwinned one are not excluded.

It is useful to note that with increasing deformation the peaks become weaker, similarly as in the experiment. This, of course, should be attributed to decreasing activity of twinning as the deformation proceeds and increasing discrepancy between the orientation of twins (or twinned subgrains in the model) created at early stages of deformation. An increasing spread of orientations around the theoretical peaks 85 and $64.4 \mathrm{deg}$ in misorientation angle distribution plots and the corresponding increasing spread around $\langle 11 \overline{2} 0\rangle$ and $\langle 10 \overline{1} 0\rangle$ directions for the misorientation axis illustrate the fact that the orientation relationship between parent and twin grain deviates from the ideal one as deformation proceeds. Recently, some experimental studies of this phenomena were performed for magnesium alloy ${ }^{[68]}$ and for titanium. ${ }^{[69]}$ One of the conclusions in the first paper was that the dislocation-twin interaction tailors the orientation of twin boundary and leads to the actual twin boundary deviating from the theoretical one. Similarly, in the second paper, it was found that the deformation may result in either increasing or lowering of the boundaries misorientation and different segments of the same grain boundary may develop different misorientation angles.

\section{DISCUSSION}

As shown in Figure 8, the model was unable to quantitatively predict the fraction of low angle boundaries. In every case, the number of low angles acquired in the simulation is lower than the measured one. This may be the result of simplifications applied in the twin-related reorientation procedure. The procedure was formulated in such a way so as to properly predict the texture of the material and to ensure the consistency between the volume fraction of reoriented grains in each metagrain and the twin volume fraction stemming from 

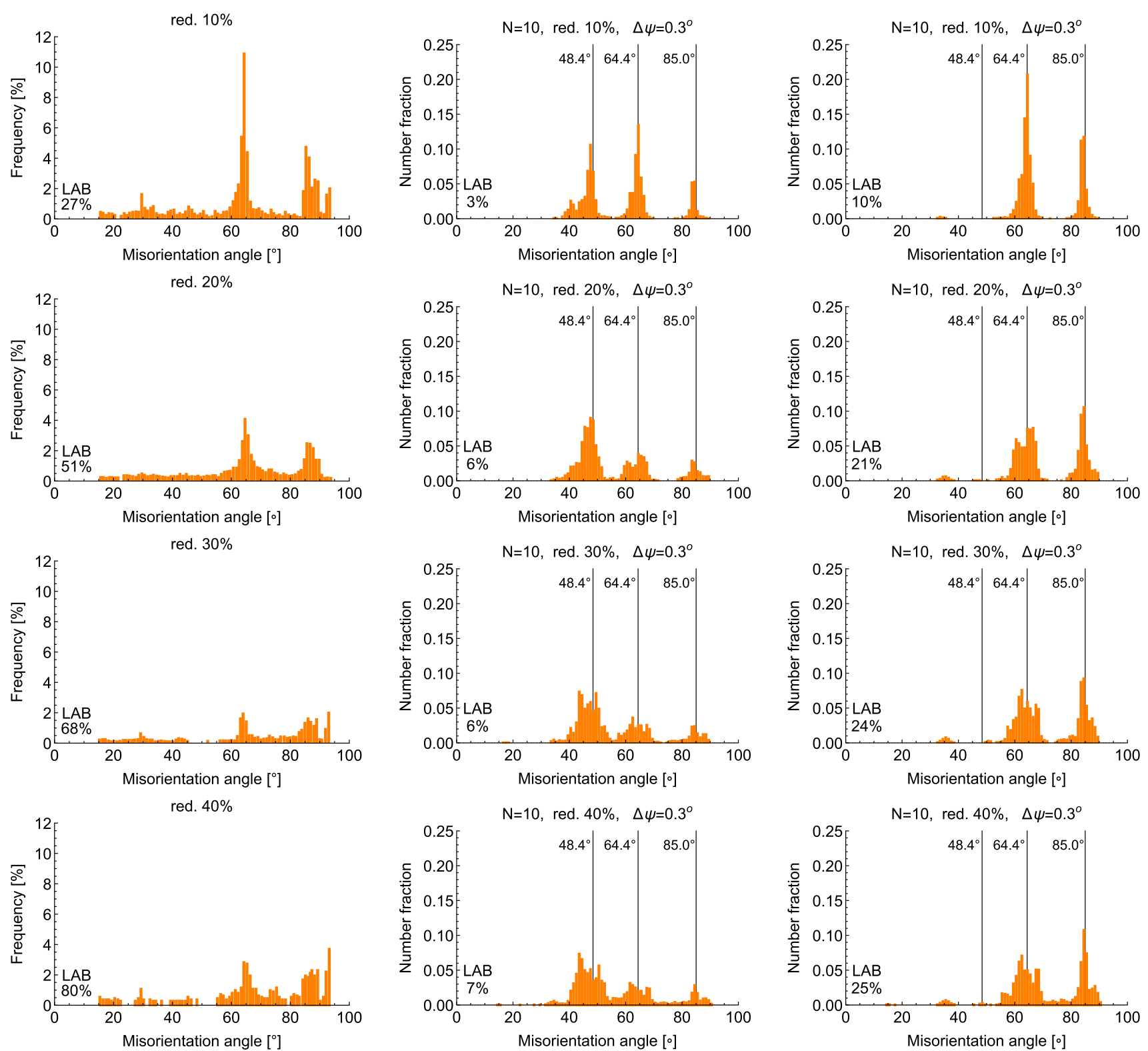

(a)

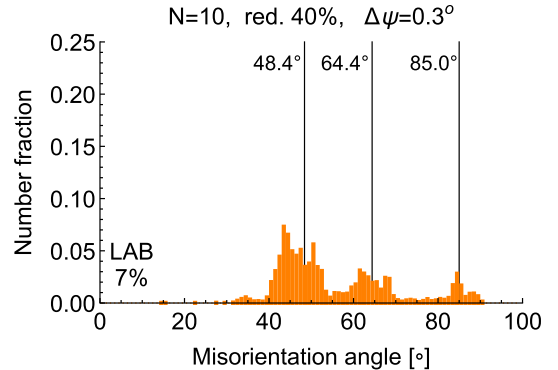

(b)

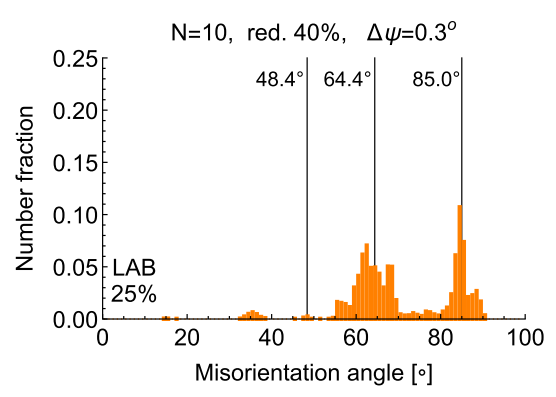

(c)

Fig. 8-The misorientation angle distribution for a sheet of CP Ti cold rolled to a total thickness reduction of 10, 20, 30, and 40 pct obtained in (a) experiment ${ }^{[42]}$ and in the simulation with the $3 \mathrm{SCP}$ model, $(b)$ for any randomly selected pair of subgrains, $(c)$ excluding the pairs composed of subgrain subject to the secondary twinning and the untwinned one. LAB stands for low angle boundary fraction.

its activity as a pseudo-slip mode. Within the procedure, the whole subgrain is reoriented when some condition is fulfilled, so that the number of subgrains within the metagrain does not increase during the simulation. In the real material, the dislocation substructure can develop inside the twin. Its development is supposed to be responsible for the high fraction of LABs present in experimental data. The $3 \mathrm{SCP}$ model is unable to predict the grain refinement inside the twins. In order to do so, the fourth level of microstructure could be added, for example assuming the composite grain model ${ }^{[54]}$ at the level of subgrain. This, however, would make the model much more numerically costly and is beyond the scope of the present paper.
Yet another option would be to apply the reorientation scheme similar to the one proposed in Reference 53, where the orientations of twins are predefined and during the simulations the volume fractions of different twin variants are measured. In such a variation, the simulation of rolling using the two-scale model with the Taylor transition could be first performed, wherefrom the evolution of volume fractions of each twin variant for every metagrain could be obtained. Then, for each metagrain, composed of subgrains of the respective variant taken in relevant evolving proportions, the simulation using the VPSC model could be performed (with a disabled primary twin mode in subgrains related to the respective variant). On this lower level, the 


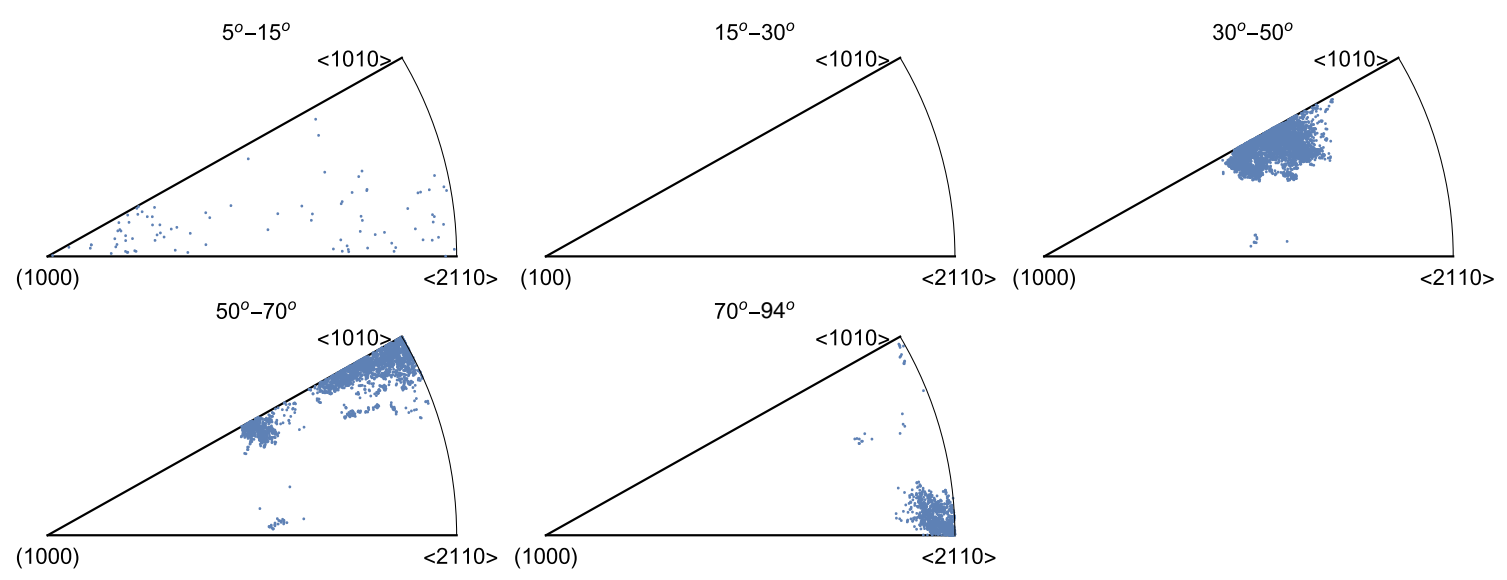

(a)
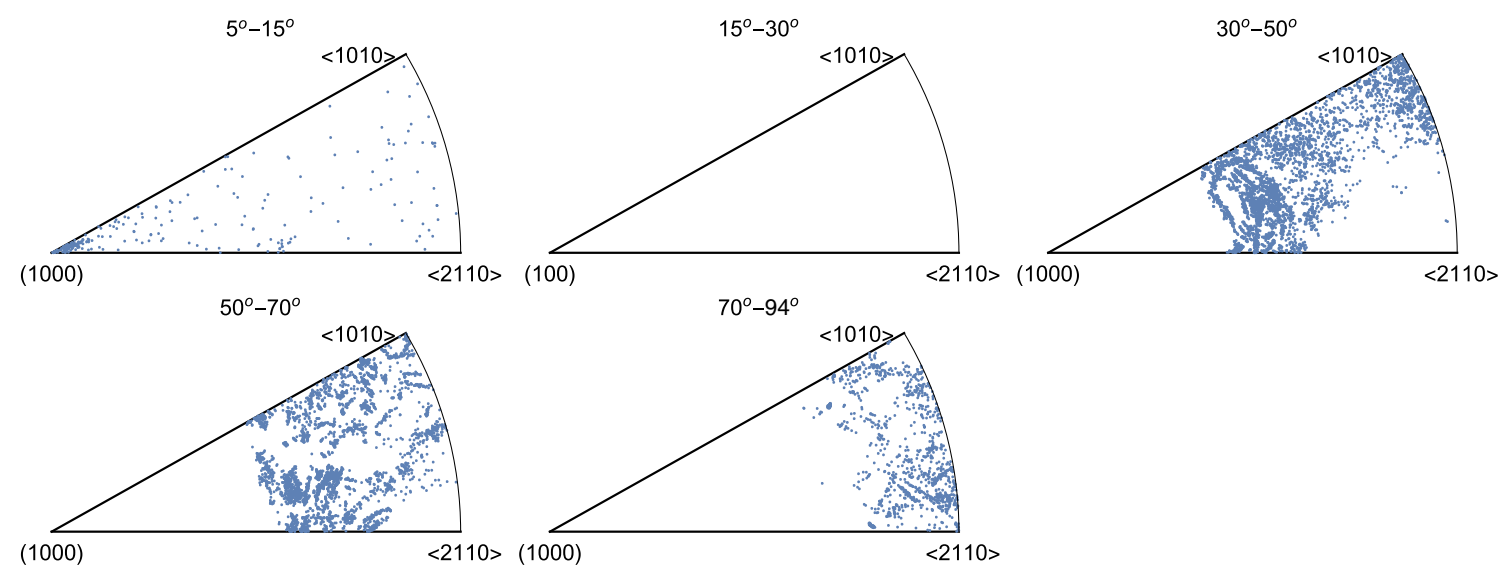

(b)

Fig. 9-Misorientation-axis distributions calculated for the randomly selected pairs of subgrains in the CP Ti rolled to reductions of $(a) 20$ pct and (b) 90 pct.

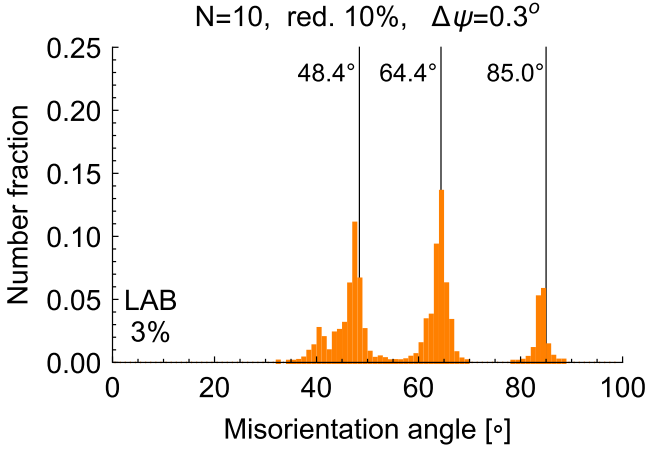

(a)

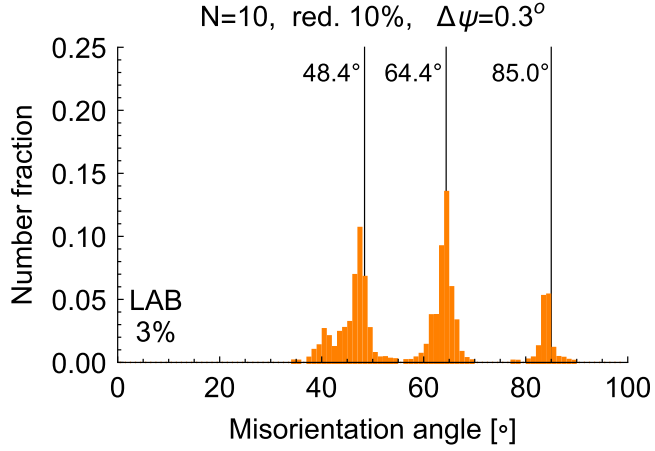

(b)

Fig. 10 - Comparison of misorientation distributions calculated for $(a) 12$ and $(b) 1$ randomly selected neighbors.

secondary twins and intra-twin refinement could be accounted for. Such procedure would be again much more computationally demanding than the one proposed in the present study, but it should be at least more efficient than the four-scale model. On the other hand, it still could not describe the possible refinement inside the secondary twins. To include such effects in this approach, the secondary twins could be also modeled during the first simulation using the Taylor model on the upper micro-macro scale, but in such a case the model would probably be more or less as costly as the four-scale model, because the number of metagrains times primary twin variants times secondary twin variants had to be accounted for. 
It is maybe important to note that in the model we do not have any information about the relative frequencies of boundaries between subgrains belonging to matrix and twins. Such information may be supplied by experiment and used in the model to improve the procedure of selecting pairs of neighbors for calculating misorientation angle distribution. Following this idea, in order to have greater insight, we have also calculated distributions of misorientation angles

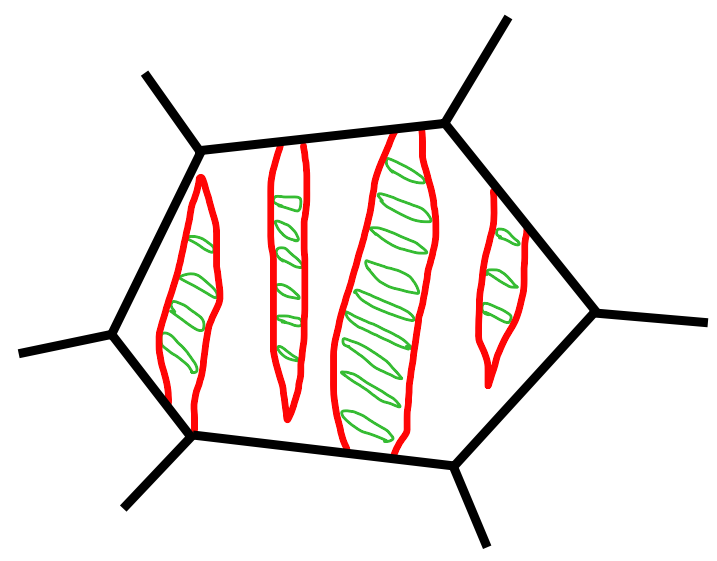

Fig. 11-The schematic view of the primary (in red) and secondary (in green) twins in single titanium grain showing that the number of boundaries between secondary twins and matrix is very low. The schematic can be compared with experimental microstructures, as shown, e.g., in Fig. 2 in Ref. [46] (Color figure online). between subgrains belonging to the same phase (matrix or a specific twin variant). In such a case, it appears that the fraction of low angle grain boundaries (misorientation angle below $15 \mathrm{deg}$ ) is about 100 pct for reductions up to 60 and about 90 pct for 90 pct reduction. To conclude, one can state that the fraction of LABs can be correctly predicted using the 3SCP model if the distributions obtained for matrix-matrix, matrix-twin and twin-twin boundaries are combined in appropriate proportions. It could be interesting to apply experimental data concerning the relative number of every type of boundary and see to what extent the results of simulation are consistent with experimental misorientation distribution. This way one could also assess the error stemming from not including the intra-twin refinement.

Figure 12 shows the misorientation distributions calculated for sets of misorientation angles as shown in Figure 8 appended by adding also the sets of misorientation angles between subgrains belonging to the same phase mentioned in the previous paragraph. It can be seen that the fraction of LABs for 10 and 20 pct thickness reductions is more or less similar to the one observed experimentally. Unfortunately, for reductions of 30 and 40 pct, the fraction of LABs is again lower than the experiment. This can be explained by rising proportion of the intra-phase boundaries to the inter-phase boundaries stemming from ongoing intraphase refinement occurring by mechanisms analogous to the ones existing in FCC materials with higher thickness reduction.
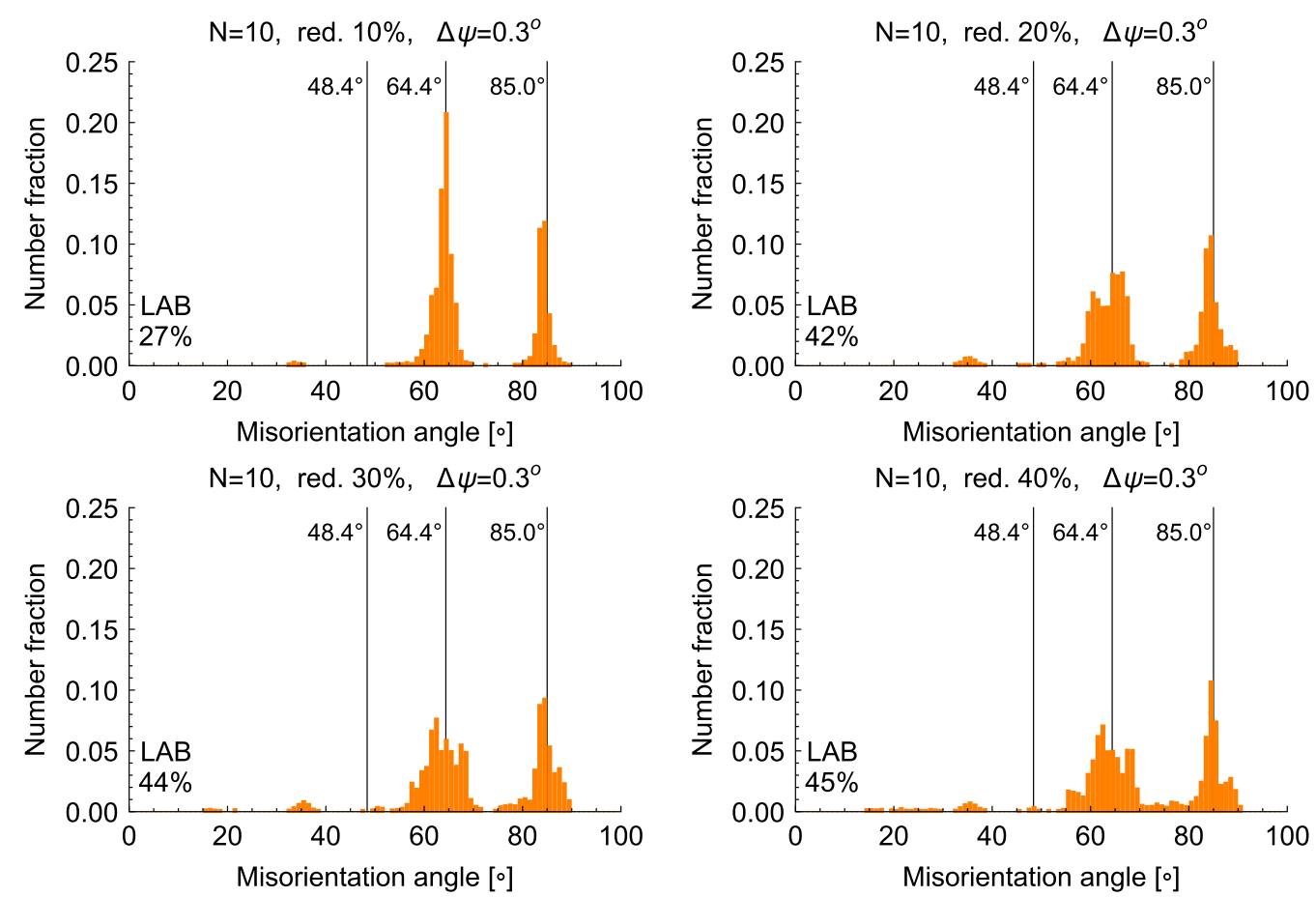

Fig. 12-The misorientation angle distribution for a sheet of CP Ti cold rolled to a total thickness reduction of 10, 20, 30, and 40 pct obtained in the simulation with the 3SCP model. The distributions are plotted by combining (in equal proportions) the sets of misorientation angles between randomly selected subgrains and angles between subgrains belonging to the same phase (matrix or a given twin variant). 


\section{SUMMARY AND CONCLUSIONS}

The aim of the paper was to examine the applicability of the recently proposed $3 \mathrm{SCP}$ model ${ }^{[8]}$ to the case of grain refinement in HCP material. Within the approach, the probabilistic twin volume consistent scheme ${ }^{[50]}$ was used to account for appearance of twin-related orientations. The simulations of texture evolution and grain refinement in $\mathrm{CP}$ Ti subjected to cold rolling were performed. The results were compared with the experimental data available in References 42 through 45. It was found that the simulated textures were in accordance with the experimental ones. Concerning the predictions of the misorientation angle distribution, the basic components of the distributions observed in experiments in the range of high angle boundaries were well identified. The peaks are associated with twinning activity. However, the application of the 3SCP model to the case of the HCP material also had difficulties, namely the fraction of low angle boundaries was underestimated by the model. Possible solutions to the problem were discussed. To this aim, some kind of a four-scale model could be developed, a different reorientation procedure can be applied, or the postprocessing of the data can be enhanced using experimental data.

To the best of the authors' knowledge, there is no model that is able to predict misorientation angle distributions in the microstructure of HCP metals, and therefore, the results presented in the paper can be considered as novel. The capabilities of the 3SCP model as compared to other grain refinement models have been summed up in Reference 8. First, contrary to the model of Leffers, ${ }^{21,22]}$ the orientations within the microstructure do not have to be predefined. This enables application of the model to study any deformation process. For example, it has been applied to study ECAP in References 8 and 48 and rolling in Reference 48 and the present paper. Second, the intra-granular strain heterogeneity is considered, contrary to models presented in References 23 through 25 . Third, contrary to References 23 through 26 the number of orientations during the simulation does not change, which increases the computational efficiency of the model. In the present analysis, it is demonstrated that those advantages are not lost when the 3SCP model is combined with PTVC reorientation scheme to account for twinning. The proposed approach provides results of statistical character. In the model, there is no information concerning the spatial distribution of subgrains in the metagrain or metagrains in the polycrystalline representative volume. This can be treated as a drawback but also as an asset because the outcomes, when a sufficiently large number of orientations are taken for the analysis, are expected to be statistically representative and not related to the specific microstructure realization.

\section{OPEN ACCESS}

This article is distributed under the terms of the Creative Commons Attribution 4.0 International License (http://creativecommons.org/licenses/by/4.0/), which permits unrestricted use, distribution, and reproduction in any medium, provided you give appropriate credit to the original author(s) and the source, provide a link to the Creative Commons license, and indicate if changes were made.

\section{APPENDIX: CONVENTIONS USED IN PLOTTING THE TEXTURES OF HCP METALS AND ALLOYS}

The stereographic projection of HCP crystals depends on the convention used for the denomination of orthogonal coordinate system. The hexagonal system is very convenient for describing the HCP crystals, due to its ability to naturally take into account the crystal symmetries. As this system is not orthogonal, it has to be transformed to the orthogonal one in order to enable the description of the lattice orientation. Two conventions for this transformation are used in the pole figure plotting software:

1. $x$ axis is parallel to $a_{i}(i=1,2,3$, the choice of $i$ is arbitrary because of the crystal symmetry), $c f$. Figure A1(a),

2. $y$ axis is parallel to $a_{i}, c f$. Figure A1(b).

$z$ axis is always parallel to the $c$ axis of the crystal, and the unspecified axis ( $y$ in convention 1 and $x$ in convention 2.) is perpendicular to both defined axes.

In order to show the significance of the choice of convention Figure A2 shows the (0001), $\{10 \overline{1} 0\}$ and $\{11 \overline{2} 0\}$ pole figures plotted for a single orientation described by Euler angles $(0,0,0)$. It is readily seen, that the choice of convention does not have any influence on the (0001) pole figure, because the " $\mathrm{c}$ " direction is the same in both conventions. The choice of the convention is however very important in case of $\{10 \overline{1} 0\}$ and $\{11 \overline{2} 0\}$ pole figures, namely it leads to their mutual interchange. As the choice of the convention is crucial for the pole figures obtained it should be made consciously and explicitly stated in the publication. Such an approach could save many misunderstanding across different groups of researchers.

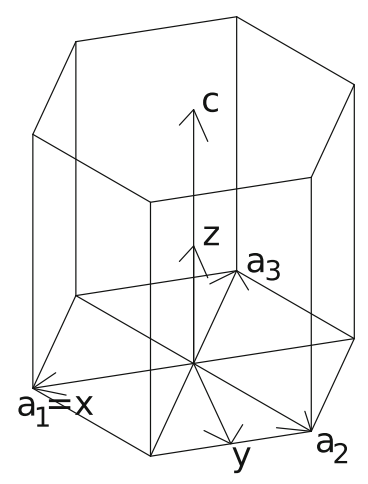

(a)

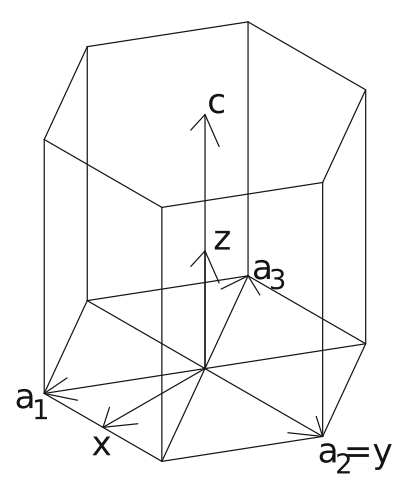

(b)
Fig. A1-Conventions used for the stereographic projection of HCP crystals, $(a)$ convention 1 and $(b)$ convention 2 . 
(0001)
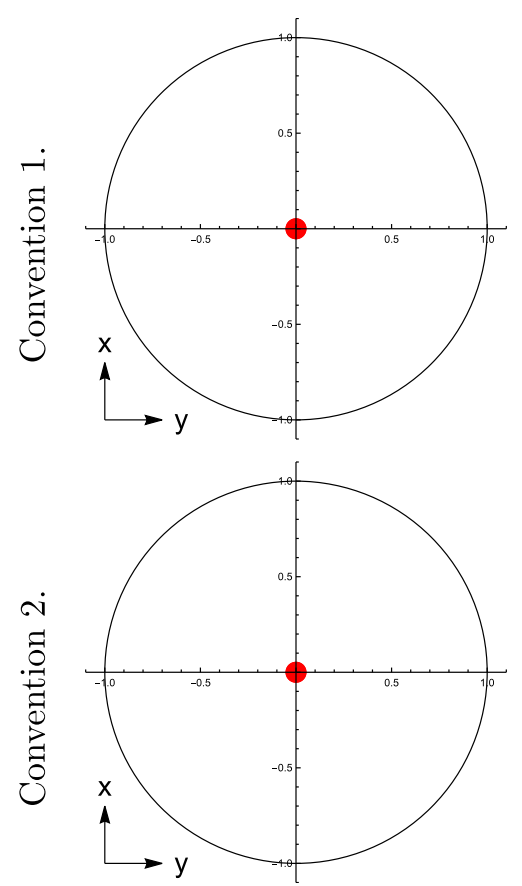

$\{10 \overline{1} 0\}$
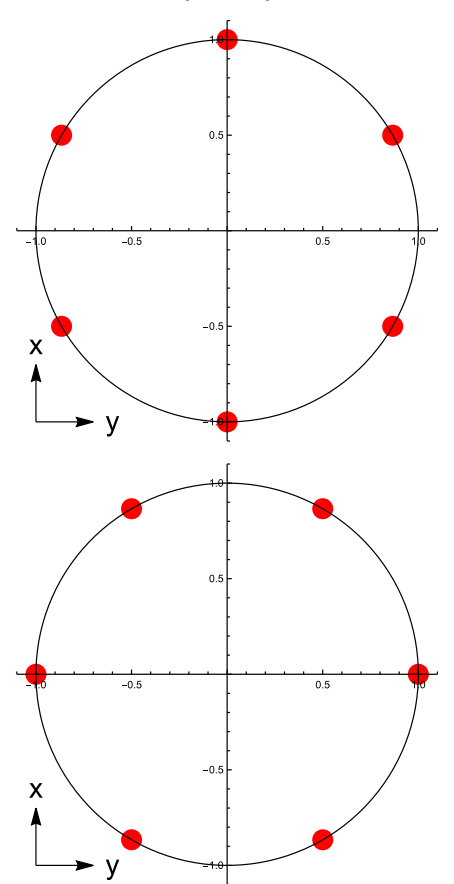

$\{11 \overline{2} 0\}$
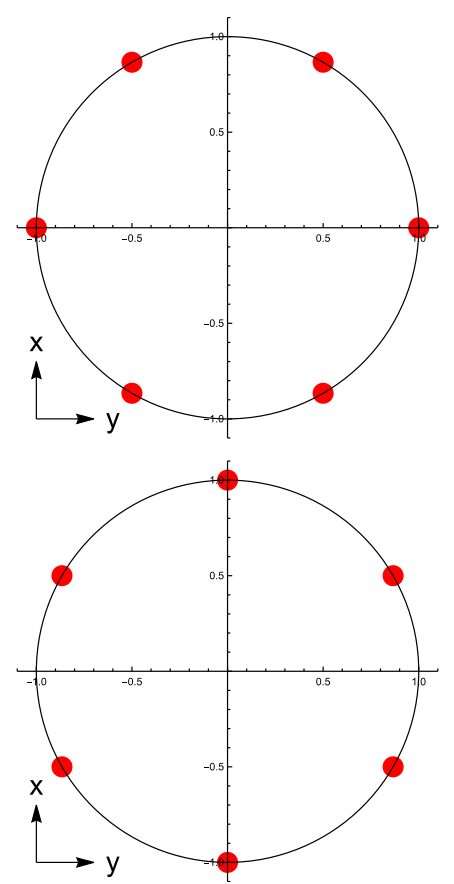

Fig. A2- $(0001),\{10 \overline{1} 0\}$ and $\{11 \overline{2} 0\}$ pole figures for $(0,0,0)$ orientation according to both conventions.

\section{REFERENCES}

1. D. Kuhlmann-Wilsdorf: Acta Mater., 1999, vol. 47, pp. 1697-1712.

2. X. Huang: Scripta Mater., 1998, vol. 38, pp. 1697-1703.

3. D.A. Hughes and N. Hansen: Acta Mater., 1997, vol. 45, pp. $3871-86$.

4. N. Hansen, X. Huang, and D.A. Hughes: Mater. Sci. Eng. A, 2001, vol. 317, pp. 3-11.

5. R.Z. Valiev and T.G. Langdon: Prog. Mater. Sci., 2006, vol. 51, pp. 881-981.

6. T.G. Langdon: Mater. Sci. Eng. A, 2007, vol. 462, pp. 3-11.

7. I.J. Beyerlein and L.S. Tóth: Prog. Mater. Sci., 2009, vol. 54, pp. $427-510$.

8. K. Frydrych and K. Kowalczyk-Gajewska: Mater. Sci. Eng. A, 2016, vol. 658, pp. 490-502.

9. A. Jäger, V. Gärtnerova, and K. Tesař: Mater. Sci. Eng. A, 2015, vol. 644 , pp. $114-20$

10. C.F. Gu, L.S. Tóth, D.P. Field, J.J. Fundenberger, and Y.D. Zhang: Acta Mater., 2013, vol. 61, pp. 3027-36.

11. B. Beausir, S. Biswas, D.I. Kim, L.S. Tóth, and S. Suwas: Acta Mater., 2009, vol. 57 (17), pp. 5061-77.

12. R.B. Figueiredo and T.G. Langdon: J. Mater. Sci., 2010, vol. 45 (17), pp. 4827-36.

13. C.W. Su, L. Lu, and M.O. Lai: Mater. Sci. Eng. A, 2006, vol. 434 (1-2), pp. 227-36.

14. M. Janeček, M. Popov, M.G. Krieger, R.J. Hellmig, and Y. Estrin: Mater. Sci. Eng. A, 2007, vol. 462 (1), pp. 116-20.

15. R. Lapovok, Y. Estrin, M.V. Popov, and T.G. Langdon: Adv. Eng. Mater., 2008, vol. 10 (5), pp. 429-33.

16. S.X. Ding, C.P. Chang, and P.W. Kao: Metall. Mater. Trans. A, 2009, vol. 40A, pp. 415-25.

17. D.K. Yang, P. Cizek, P.D. Hodgson, and C.E. Wen: Acta Mater., 2010, vol. 58 (13), pp. 4536-48.

18. Z. Zeng, S. Jonsson, and H.J. Roven: Acta Mater., 2009, vol. 57 (19), pp. 5822-33.

19. Y Beygelzimer: Mech. Mater., 2005, vol. 37, pp. 753-67.

20. H. Petryk and S. Stupkiewicz: Mater. Sci. Eng. A, 2007, vol. 444, pp. 214-19.

21. T. Leffers: Int. J. Plasticity, 2001, vol. 17, pp. 469-89.

22. T. Leffers: Int. J. Plasticity, 2001, vol. 17, pp. 491-511.
23. I.J. Beyerlein, R.A. Lebensohn, and C.N. Tomé: Mater. Sci. Eng. A, 2003, vol. 345, pp. 122-38.

24. A.A. Nazarov, N.A. Enikeev, A.E. Romanov, T.S. Orlova, I.V. Alexandrov, I.J. Beyelein, and R.Z. Valiev: Acta Mater., 2006, vol. 54, pp. 985-95.

25. N.A. Enikeev, M.F. Abdullin, A.A. Nazarov, and I.J. Beyerlein: Int. J. Mater. Res., 2007, vol. 98, pp. 167-71.

26. L.S. Tóth, Y. Estrin, R. Lapovok, and C. Gu: Acta Mater., 2010, vol. 58 , pp. $1782-94$.

27. M. Ortiz and E.A. Repetto: J. Mech. Phys. Solids, 1999, vol. 36, pp. 286-351.

28. H. Petryk: C. R. Mecanique, 2003, vol. 331, pp. 469-74.

29. H. Petryk and M. Kursa: J. Mech. Phys. Solids, 2013, vol. 61, pp. 1854-75.

30. H. Petryk and M. Kursa: Int. J. Numer. Methods Eng., 2015, vol. 61, pp. 1854-75.

31. D. Raabe, Z. Zhao, and W. Mao: Acta Mater., 2002, vol. 50 (17), pp. 4379-94.

32. S.J. Park, H.N. Han, K.H. Oh, D. Raabe, and J.K. Kim: Mater. Sci. Forum (Switzerland), vol. 408, pp. 371-76.

33. P.D. Wu, Y. Huang, and D.J. Lloyd: Scripta Mater., 2006, vol. 54 (12), pp. 2107-12.

34. M. Knezevic, B. Drach, M. Ardeljan, and I.J. Beyerlein: Comput. Methods Appl. Mech. Eng., 2014, vol. 277, pp. 239-59.

35. K. Frydrych and K. Kowalczyk-Gajewska: Unpublished Research, 2018.

36. D.-F. Zhang, H.-J. Hu, F.-S. Pan, M.-B. Yang, and J.-P. Zhang: T. Nonferrous Met. Soc., 2010, vol. 20 (3), pp. 478-83.

37. M.Z. Gzyl, A. Rosochowski, A. Milenin, and L. Olejnik: Comput. Methods Mater. Sci., 2013, vol. 13 (2), pp. 357-63.

38. H. Ding and Y.C. Shin: In ASME 2011 International Manufacturing Science and Engineering Conference, American Society of Mechanical Engineers, pp. 89-98.

39. Y. Estrin, L.S. Tóth, A. Molinari, and Y. Bréchet: Acta Mater., 1998, vol. 46, pp. 5509-22.

40. L.S. Tóth, A. Molinari, and Y. Estrin: J. Eng. Mater. Technol., 2002, vol. 124 (1), pp. 71-77.

41. H. Ding, N. Shen, and Y.C. Shin: J. Mater. Proc. Technol., 2012, vol. 212 (5), pp. 1003-13.

42. Y.B. Chun, S.H. Yu, S.L. Semiatin, and S.K. Hwang: Mater. Sci. Eng. A, 2005, vol. 398 (1), pp. 209-19. 
43. N. Bozzolo, N. Dewobroto, H.R. Wenk, and F. Wagner: J. Mater. Sci., 2007, vol. 42 (7), pp. 2405-16.

44. Y. Zhong, F. Yin, and K. Nagai: J. Mater. Res., 2008, vol. 23 (11), pp. 2954-66.

45. S.V. Zherebtsov, G.S. Dyakonov, A.A. Salem, S.P. Malysheva, G.A. Salishchev, and S.L. Semiatin: Mater. Sci. Eng. A, 2011, vol. 528 (9), pp. 3474-79.

46. S.V. Zherebtsov, G.S. Dyakonov, A.A. Salem, V.I. Sokolenko, G.A. Salishchev, and S.L. Semiatin: Acta Mater., 2013, vol. 61 (4), pp. 1167-78.

47. R.A. Lebensohn and C.N. Tomé: Acta Metall. Mater., 1993, vol. 41 , pp. 2611-24.

48. K. Frydrych: Modelowanie ewolucji mikrostruktury metali o wysokiej wytrzymałości właściwej w procesach intensywnej deformacji plastycznej (Modelling the microstructure evolution of metals and alloys of high specific strength subjected to severe plastic deformation processes), Ph.D. thesis, in Polish, Warsaw, Poland, 2017.

49. A. Morawiec: Orientations and Rotations. Computations in Crystallographic Textures, Springer, Berlin, 2004.

50. K. Kowalczyk-Gajewska: Eur. J. Mech. Solids A, 2010, vol. 29, pp. $28-41$.

51. P. Van Houtte: Acta Metall., 1978, vol. 26, pp. 591-604.

52. K. Kowalczyk-Gajewska: Comput. Methods Mater. Sci., 2013, vol. 13 (4), pp. 436-451.

53. S.R. Kalidindi: J. Mech. Phys. Solids, 1998, vol. 46, pp. 267-90.

54. G. Proust, C.N. Tomé, and G.C. Kaschner: Acta Mater., 2007, vol. 55 , pp. $2137-48$.

55. R.A. Lebensohn, H. Uhlenhut, C. Hartig, and H. Mecking: Acta Mater., 1998, vol. 46, pp. 229-36.
56. S. Dancette, L. Delannay, K. Renard, M.A. Melchior, and P.J. Jacques: Acta Mater., 2012, vol. 60, pp. 2135-45.

57. M. Homayonifar and J. Mosler: Int. J. Plasticity, 2012, vol. 28, pp. $1-20$

58. R. Hill and J.R. Rice: J. Mech. Phys. Solids, 1972, vol. 20, pp. 401-13.

59. R.J. Asaro: J. Appl. Mech., 1983, vol. 50, pp. 921-34.

60. K. Kowalczyk-Gajewska, K. Sztwiertnia, J. Kawałko, K. Wierzbanowski, K. Wroński, K. Frydrych, S. Stupkiewicz, and H. Petryk: Mater. Sci. Eng. A, 2015, vol. 637, pp. 251-63.

61. K. Kowalczyk-Gajewska: IFTR Rep., 2011, vol. 1 (2011), pp. 1-299.

62. A.A. Salem, S.R. Kalidindi, and S.L. Semiatin: Acta Mater., 2005, vol. 53, pp. 3495-02.

63. GI Taylor: J. Inst. Met., 1938, vol. 62, pp. 307-24.

64. A. Molinari, G.R. Canova, and S. Ahzi: Acta Metall., 1987, vol. 35 , pp. 2983-94.

65. C. N. Tomé and R. A. Lebensohn: Manual for Code Visco-Plastic Self-Consistent (VPSC), Version 7b. Tech. Rep., Los Alamos National Laboratory, 2007.

66. I. Karaman, H. Sehitoglu, A.J. Beaudoin, Y.I. Chumlyakov, H.J. Maier, and C.N. Tomé: Acta Mater., 2000, vol. 48, pp. 2031-47.

67. G.S. Dyakonov, S. Mironov, S.V. Zherebtsov, S.P. Malysheva, G.A. Salishchev, A.A. Salem, and S.L. Semiatin: Mater. Sci. Eng. $A, 2014$, vol. 607 , pp. $145-54$.

68. J. Zhang, G. Xi, X. Wan, and C. Fang: Acta Mater., 2017, vol. 133 , pp. $208-16$.

69. G. Salishchev, S. Mironov, S. Zherebtsov, and A. Belyakov: Mater. Charact., 2010, vol. 61 (7), pp. 732-39. 